\title{
Cognitive Aspects of Spatial Orientation
}

\section{Miroslav Macik}

Department of Computer Graphics and Interaction

Faculty of Electrical Engineering, Czech Technical University in Prague

Karlovo nam. 13, Praha 2

Email: macikmir@fel.cvut.cz

\begin{abstract}
This manuscript focuses on cognitive aspects of spatial mental modeling. We examine possibilities for merging methods for sensing and modeling of cognitive capabilities and cognitive styles with the concept of cognitive infocommunications. Related aspects of cognitive psychology, the theory of senses, sensory substitution, and mental modeling are discussed. We illustrate practical impact of emerging CogInfoCom methods on people with special needs, in particular, those with vision impairment.
\end{abstract}

Keywords: cognition; senses; cognitive infocommunications; navigation; orientation

\section{Introduction}

In the field of Cognitive Psychology, development factors and communication among different cognitive entities are essential. The representation coding corresponds not only with ways of how humans communicate using their senses but also with the way how people store the information in their brains. The sensory modality corresponds to the way humans communicate and with spatiotemporal orientation. Also, the sensory modality might affect the information coding humans use to build mental models of the environment around them.

The field of Cognitive Infocommunications (CogInfoCom) is a multi-field discipline involving among others Cognitive Psychology. According to the definition in [1] "CogInfoCom investigates the link between the research areas of infocommunications and cognitive sciences, as well as the various engineering applications which have emerged as a synergic combination of these sciences." The primary goal is to investigate how cognitive processes can co-evolve with infocommunications devices. The blending of cognitive capabilities of natural and artificial systems may result in a synergically more effective combination both on a theoretical and engineering application level. 
From the cognitive capability perspective, CogInfoCom defines two types of communication: Intra-cognitive communication between two entities with equivalent cognitive capabilities and inter-cognitive communication between entities that differ in their cognitive capabilities. From the sensor (sensory) perspective, there is sensor sharing communication, and sensor bridging communication. The other aspect is the information representation. There is representation sharing communication and representation bridging communication.

Individuals use different strategies to create and maintain mental models of the spatial environment around them. The research on mental spatiotemporal modeling of the external world emerges from Cognitive Psychology. Better comprehension of these processes in the framework of cognitive infocommunications could lead to a better concept of spatial orientation by employing blended natural and artificial capabilities. User groups with specific needs, among others visually impaired individuals, will benefit from the outcomes.

The strategies of spatial mental modeling differ between individuals. For instance, visually impaired people require specific knowledge about the environment, and they build mental models that can differ from the general population. By supporting the process of spatial orientation using info-communication technologies, we can facilitate the ability of independent navigation in the indoor and outdoor environment. This intent requires knowledge of individual cognitive capacities, spatial mapping strategies and terms a particular person uses for mental representation of objects relevant to spatial orientation.

Becoming visually impaired can be a life-changing experience and is likely to have far-reaching consequences for the person affected [2]. Loss of vision is also often associated with a psychological reaction such as depression, low morale, or poor self-esteem. Assistive devices can have a positive impact on disability and depression of those affected. However, Horowitz et al. in [3] were able to find significant evidence only for improving depression symptoms by optical compensation aids that enable their users to continue using their remaining sight and habits rather than using sensory-bridging compensation aids such as talking books. In [4], Macik et al. describes a qualitative study of everyday needs of visually impaired older adults living in a residential care institution from the perspective of the use of infocommunications. The results motivate us to employ CogInfoCom to support spatial orientation of people with special needs.

Wobbrock et al. in [5] introduced the concept of Ability based design. This concept encourages assistive technologies designers to divert their focus from disabilities to each individual's specific abilities. Authors define terms adaptable and adaptive systems. While an adaptable system can be manually adjusted to reflect specific individual needs and support her/his abilities, an adaptive system can automatically sense and model these specific individual needs/abilities and consequently perform the adaptations automatically. 
In [6], Um argues that the information society paradigm is moving towards a cyber-physical system (e.g., self-driving car) society. From the perspective of spatial information science, he leverages the importance of spatial information and its distribution. For purposes of supporting spatial mental modeling, having access to precise and up-to-date spatial information about the desired physical or virtual environment is vital. Authors of [7] argue that having actual information about the sidewalk network is necessary for the technological support of visually impaired navigation in the city. Projects exist [8] that use crowdsourcing together and maintain such information, but professional support from a big market player is often necessary.

In this paper, we aim to direct the research of CogInfoCom to explore the use of infocommunications to facilitate spatial understanding. Info-communication technologies are already being used to help users with various cognitive abilities to navigate in the indoor and outdoor environment. Several projects are aiming to support visually impaired, wheelchair users, but also people with mental challenges in their tasks related to indoor and outdoor navigation and spatiotemporal orientation. Examples of such projects are detailed in Section 3.

In the domain of CogInfoCom, the spatiotemporal orientation has been already addressed by numerous works. Macik et al. in [9] propose an indoor navigation system that does not depend on complex devices carried by users. Instead, several navigational terminals guide the visitors to their destination. Specific aspects of the orientation of older adults with vision impairment are discussed in [4]. An indoor surveillance system that supports spatial orientation is addressed in [10]. In [11], authors focus on localization of visually impaired in the outdoor environment. Ito et al. in [12] propose a cognitive model of sightseeing for a mobile support system. Kutikova et al. investigate ICT used in travel-related activities of wheelchair users. Sik et al. in [13] describe an implementation of a geographic information system that connects different data sources.

This paper is structured as follows. In Section 2, we discuss the scientific foundations for spatial mental modeling. We put particular focus on selected aspects of Cognitive Psychology, mental modeling and the theory of senses. In Section 3, we list relevant approaches that aim to support orientation in the space environment, spatial mental modeling or use a special method for sensory substitution. Section 4 concludes the paper, it discusses how theoretical foundation described in the paper can contribute to the future research in the field of CogInfoCom.

\section{Background}

In this section, we discuss the scientific foundations for spatial mental modeling within the framework of CogInfoCom. Firstly, we define the basic terms related to 
the mental mapping and exploration of spatial environments. This is followed by a survey of psychological aspects relevant to the navigation and orientation of the visually impaired. Finally, we provide a survey on sensory modalities in relation to their utility for the purposes of spatial orientation.

According to the Oxford dictionary [14], "Navigation is the process or activity of accurately ascertaining one's position and planning and following a route." Wayfinding has exactly the same definition in that dictionary.

For our purposes, we define term Navigation System as an artificial entity that helps with one's navigation through the physical or virtual spatial environment. The Navigation System maintains information about one's target destination and by inter-cognitive communication provides Navigation Instructions to reach the destination.

"Orientation is the action of orienting someone or something relative to the points of a compass or other specified positions" [15]. An Orientation System is an artificial entity that provides Orientation Cues to help maintain one's orientation in physical or virtual space environment. Orientation System helps to maintain one's Situational awareness [16].

\subsection{Cognition, Cognitive Psychology, Cognitive Science and Artificial Intelligence}

According to Oxford the dictionary [17], "cognition is the mental action or process of acquiring knowledge and understanding through thought, experience, and the senses". While cognitive psychology focuses on studying mental processes of humans, Cognitive science is according to [18] "the interdisciplinary study of mind and intelligence, embracing philosophy, psychology, artificial intelligence, neuroscience, linguistics, and anthropology" Artificial intelligence (AI) involves the study of cognitive phenomena in machines. Another scientific field that investigates phenomena that affect the ways people represent information for purposes of communication and memory is linguistics [19]. According to the Oxford dictionary, linguistics is the scientific study of language and its structure, including the study of grammar, syntax, and phonetics.

From the perspective of cognitive psychology, high cognitive diversity among humans is possible. Several factors can affect the way we think, including, genetics, developmental factors, culture, and environmental factors in general. Cognitive psychologists suggest various cognitive styles - categorization of individual ways of thinking from multiple perspectives. Cognitive styles bridge cognition and personality. From the cognitive perspective (cognitive centered approaches), individuals can be assessed on the scale of reflection-impulsivity [20], while Witkin is his theory of field dependency [21] examines one's tendency to rely on information provided by the outer world. Based on the perspective from 
which an individual represents information Richardson [22] suggests two styles visualizer and verbalizer.

Another dimension worth considering is, perspective is the personality (personality centered approaches). Foundations of personality centered approaches stem from Jung's cognitive styles [23]. In his approach, individuals can be characterized as differing in terms of two attitudes (extraversion and introversion), two perceptual functions (intuition and sensing), and two judgment functions (thinking and feeling). The popular Myers-Briggs Type Indicator (MBTI) [24] is based on Jung's approach. Gregorc's energic model [25] proposes that cognitive styles can reflect two basic dimensions: use of space (concrete or abstract) and use of time (random or sequential).

Sternberg et al. [26, 27] proposed the theory of mental self-government. The basic idea behind the theory is that the various styles of public government may be external reflections of the styles that can be found in the mind. Also, the authors argue that in contrast to previously described approaches for cognitive styles, everyone possesses every style to some degree. Cognitive styles seem to be largely a function of an individual's interactions with tasks and situations. A person with one style in one task or situation may have a different style in another task or situation.

In [28], Torok describes the estimated transition from human-computer interaction to cognitive infocommunications from the perspective of cognitive science. He sees the development in ways how the new systems will understand humans. The new forms of interaction should reflect an understanding of human behavior, human limits, needs and ultimately human cognition. Although the goal of fully understanding human cognition cannot be reached in the foreseeable future, as the artificial cognitive system must have been able to comprehend how an individual thinks in the full extent.

\subsection{Psychological Aspects of the Spatial Orientation of the Visually Impaired}

Cognitive psychologists have three basic theories about the spatial cognition of the visually impaired. Ungar [29] mentions theories of deficiency, inefficiency and difference. The theory of deficiency presumes that the lack of visual experience results in a complete lack of spatial understanding. The inefficiency theory assumes that spatial abilities of visually impaired individuals are similar to (but less efficient than) those of sighted people. The last difference theory assumes that a vision impairment may result in abilities which are qualitatively different but functionally equivalent to abilities of sighted people. The empirical research disproved the theory of deficiency as experiments proved that congenitally blind individuals have the spatial understanding. 
Spatial schemes are used in abstract thinking [30, 31]. Human memories are anchored in relationship to places; spatial metaphors help to structure our memory. In the case of sighted people, vision is the primary modality supporting spatial orientation. Sight provides reliable and rich information about objects in space. Moreover, these objects do not need to be in the close distance. Although hearing can also provide information about a distant object, the quality and reliability of this input is significantly lower than in case of sight. Also, sight can simultaneously provide information about multiple objects (central and peripheral vision).

Even in the case of sighted people, the visual stimuli are not the only ones used for coding spatial information. Typically, mental representations are based on overlapping information from more sensory modalities [31, 32]. Besides vision, spatial cognition employs hearing, spatial orientation stimuli from the vestibular system and kinesthetic stimuli from proprioceptors. Thinus-Blanc and Gaunet in [33] show evidence that representation of space in blind persons differs and that lack of visual stimuli has critical and irreversible effects at the level of brain function. However, results of studies (e.g., [34]) investigating spatial orientation of visually impaired show no or only slight performance decline of congenitally blind individuals. The empirical results support the difference theory, and the probable cause is that many blind persons have developed highly effective spatial strategies.

Kitchin et al. in [35] present a literature overview of research related to understanding spatial concepts by visually impaired individuals. The authors argue that further research is necessary to fully understand how visually impaired people orientate themselves in spatial environments defined at a geographic scale rather than in small-scale artificial environments evaluated in laboratory studies.

Navigation of visually impaired primarily employs hearing, touch, kinesthetic and olfactory stimuli. The information provided by these senses has lower reliability and lacks the possibility of continuous simultaneous sensing of multiple spatial reference objects. This leads to the use of different spatial references and mental coding of spatial information by those blind and visually impaired. Consequently, the navigation strategies also differ. According to [31], this plays a significant role in demands on the capacity of working memory.

\subsection{Cognitive Maps of Spatial Environment}

Siegel and White [36] define three types of spatial knowledge - landmark, route, and survey. Landmarks are specific geographic locations, strategic places to which a person travels (e.g., shop, church, bus stop). Routes correspond to a sequence of landmarks. Survey (configurational) knowledge correspond to a map-like representation of space environment. 
According to Brock et al. [37], preparation in a safe environment like home using tactile maps can provide visually impaired with cognitive maps of the environment they intend to visit and consequently help them to overcome fear related to traveling. Tactile map reading is not intuitive and must be learned. It implies several challenges for the inexperienced map user.

Loomis, Klatzky and Giudice in [38] investigate spatial representations of threedimensional space in spatial working memory. Authors call representations in the short-term working memory spatial images. These representations differ from representations in the long-term memory and from percepts (perceptual representations). An individual can perform mental manipulations of spatial images such as relative parallax (caused by own movement in the space). Spatial image is multisensory in origin. It can be instantiated in spatial working memory by visual, auditory and haptic stimulation and by spatial language. Also, Spatial images can be instantiated by recall from long-term memory. There is research evidence that spatial mental images are amodal.

Lahav and Mioduser in [39] investigate the construction of cognitive maps of unknown spaces using a multi-sensory virtual environment for people who are blind. The authors present a study that investigates the creation of spatial cognitive map using compensatory sensory channels within multi-sensory virtual environment simulating real target space. The goal is to assist visually impaired in their anticipatory exploration and cognitive mapping of unknown spaces. Results of their study provide strong evidence that exploration of multi-sensory virtual environment provides a robust foundation for the development of comprehensive cognitive maps of unknown space. The virtual environment supports two modes teacher mode and learning mode. In the teacher mode, environment editor can be used to specify the model of the spatial environment, force feedback effects, and audio feedback. In the learning mode, a force-feedback joystick is used to explore the virtual environment. The results show that in the virtual environment the participants get more holistic and comprehensive cognitive maps that by exploration of the real environment. In subsequent research [40], the authors investigate the integration of multi-sensory virtual environment into a rehabilitation program to improve orientation and mobility skills for people who are blind. This research has shown the positive effect of a virtual reality environment in the subsequent navigation and orientation in unfamiliar space.

Papadopoulos, Koustriava and Barouti in and [41] study the ability of visually impaired to create cognitive maps of familiar and unfamiliar spaces. Authors compare cognitive maps created through audio-tactile maps and through walked experience in terms of precision and inclusiveness. Results of a study with thirty visually impaired participants support the usefulness of tactile maps for the creation of spatial knowledge. Similarly to audio-haptic virtual environment [39], exploration of audio-tactile maps provides more complete cognitive maps than walking along a route in an unfamiliar area. 
Kitchin and Jacobson in [42] present a survey of techniques to collect and analyze data on how visually impaired learn, understand and think about geographic space. In their review, they divided tests to those that measure aspects of route knowledge and those that measure aspects of configurational (survey) knowledge. The authors point to issues of validity of some studies. Small sample sizes cause the biggest concerts. Furthermore, there are many studies assessing respondents' knowledge of micro-scale artificial environment rather than real-world macrospaces people deal with in real life.

Loomis, Klatzky, and Golledge in [34] summarize results of basic research related to navigation and orientation of visually impaired. On the basis of these results, they propose navigation system utilizing global positioning system (GPS), geographical information systems (GIS) and virtual acoustics. There are two distinct means of keeping track of position while traveling: landmark-based navigation and path-integration. In landmark-based navigation, senses provide a traveler with information about current position relative to a landmark, often in conjunction with an external map or cognitive map. In path integration, the traveler uses sensed motion to upgrade the current position and orientation relative to some starting point. There are no major differences in patch integration ability among blind and sighted individuals. Concepts described in this paper resulted in proposing the concept of spatial images described above.

Kacorri et al. [43] focus on environmental factors affecting indoor navigation of visually impaired. They present a study based on analysis of real-world trajectories. They identified relationships between deviation from the optimal route and trajectory variability. Furthermore, navigation performance is affected by elements of the environment, route characteristics, localization error, and instructional cues that users receive. Most studies related to navigation and orientation of visually impaired does not consider environmental factors to a sufficient extent.

Izso [44] investigates the benefits that CogInfoCom based assistive technologies can offer to individuals with non-standard cognitive characteristics. In his paper, he considers visually impaired, deaf, and individuals with a mental disorder (depression, bipolar affective disorder, schizophrenia, dementia, and developmental disorders like autism). Assistive technologies can increase the independence of individuals with non-standard cognitive characteristic by enabling them to perform tasks that they were formerly unable to accomplish. On the basis of properties of the human cognitive system, the impairments can be caused by issues of sensory sub-system (vision, hearing, smell, touch, and proprioception), issues of pre-processing system (sensory register, attention), memory problems, or impairments of higher cognitive functions (problem solving, reasoning, language). The concept of ability-demand gap correlated the level of personal abilities to perform a certain task and ability demand required by the task in a particular context. The gap between an individual's abilities and the demand can be called a handicap. An impairment does not necessarily lead to a handicap. 
The individual's abilities are either sufficient to carry out a particular task, or appropriate assistive technology can be employed to cover the gap.

When considering cognitive consequences of limitations of other senses than vision, we can mention work by Esposito et al. In [45], they examine differences between hearing and deaf subjects in decoding foreign emotional faces. They investigated the ability of deaf and hearing individuals to correctly label foreign faces expressing six basic emotions of happiness, sadness, surprise, anger, fear, and disgust. The presented study focused on comparing the ability of Italian deaf and hearing subjects in decoding Dutch facial emotional expressions. The results show that deaf individuals performed significantly poorly in decoding accuracy and intensity of disgust, surprise, and anger. There are also indications that emotional experience related to culture affect the performance of identifying facial expressions of representatives of another culture.

\subsection{Senses and Sensory Substitution}

Experimental results support theories about better performance of blind and visually impaired individuals in case of hearing recognition. In the case of touch, the results are mixed and correspond to a particular task. Blind and Visually impaired show better performance in recognition of fine textures and basic shapes. Goldreich in [46] showed that passive tactile acuity is significantly better in the case of blind subjects than in the case of sighted subjects. Results showed that the average blind subject had the acuity of an average sighted subject of the same gender but 23 years younger. The acuity is dependent on the force of contact between the stimulus surface and skin, declines with subject age, and is better in the case of the woman than men.

There are different neurophysiological reasons for the superior performance of visually impaired in tasks related to other senses than vision. A cross-modal reorganization is possible for brain regions originally related to sight. These regions are colonized by touch and healing thanks to the mechanism of synaptic plasticity [31, 47, 48]. Empirical proof for this fact is the activation of visionrelated areas of the brain by tactile, auditory and olfactory stimuli in case of congenitally blind subjects.

Loomis et al. in [49] describe various approaches for sensory substitution of vision from the perspective of cognitive science and neuroscience. There are clear constraints on the utility of new sensory substitution technologies that stem from properties of perceptual and cognitive processing. From the application perspective, there are general-purpose and special-purpose sensory substitution aids. For instance, distal attribution (experiencing tactile stimulation on the skin surface of an object external to the user) has been studied for more than three decades, but no general-purpose vision-to-touch sensory substitution method robust enough to be used in practical life emerged. 
Vision can be substituted by other spatial senses - hearing and touch. From the perspective of channel bandwidth, vision outperforms the other two senses. Therefore, direct translation of visual information provided for instance by camera sensor inevitably leads to loss of information. There is a smaller effective field of view for touch. It can be caused by lower working memory available for touch processing. Also, figural processing is associated with visual perception and is less accessible by touch.

From the perspective of CogInfoCom, all these technologies provide sensory bridging, but some of them provide also representation bridging communication. Loomis et al. in [49] suggest that methods employing representation bridging can be more useful for visually impaired as they can reflect differences in cognitive processing of stimuli provided by different senses. Effective sensory substitution (sensory bridging) is likely to depend on more substituting senses and on meaningful representation bridging and filtering.

The process of keeping mental track of directions and distances of previously viewed objects is called mental updating. In [50] Bennett et al. shows that performance in this process is decreasing with age. There are two theoretical models of spatial updating allocentric (all locations, including that of navigation, are designated in terms of extrinsic coordinates) and egocentric (the origin is centered on the navigator and external locations are updated accordingly).

\subsubsection{Touch}

Haptic modality has great significance for spatial orientation of the visually impaired. It can be employed in the exploration of the near environment reachable by touch. Assistive aids, most importantly the white cane, are used to extend the area that can be efficiently explored by touch. In survey [51] Csapo et al. describe haptic interaction as exploration based on recognition through touching, grasping or pushing/pulling movements. The convention refers tactile perception to an interaction where sensations are obtained through the skin, while the haptic perception extend tactile perception with impressions received through the muscles, tendons, and joints. The authors conclude that the amount of information that can be provided using tactile and haptic feedback is lower than through the visual and auditory senses. In [52] Loomis and Lederman present a survey of fundamental research related to the modality of touch. Similar to [51], they stated that touch comprises two distinct senses - the cutaneous sense (tactile perception) and kinesthesis. The haptic perception involves both cutaneous and kinesthetic stimuli. Touch is segmented and sequential, there are great demands on memory.

In [53] Holloway et al. compared accessible tactile maps with 3D models. The experiments indicated better performance of 3D models in short-term recall and understandability and their usefulness for orientation and mobility training of visually impaired. Haptic exploration is the process of exploring an object by touch. It requires significant cognitive effort. Different movements are required 
for perceiving different aspects of an object. Lateral movement is convenient for getting information about the texture, the enclosure for global shape and contour following is necessary for sensing exact shape.

Brock et al. [37] present a comparative study of a classical tactile raised-line map and an interactive map composed by a multi-touch screen, raised-line overlay, and audio output. Visually impaired individuals use tactile relief maps are used to acquire the mental representation of space. Results show that replacing braille labels with simple audio-tactile representation improved efficiency and user satisfaction. Also, long-term evaluation of spatial information acquired from tactile maps is suitable to build robust survey-type mental representation in visually impaired users.

Aasen and Nærland in [54] investigated responses to verbal and tactile requests to children with the combination of congenital blindness, and intellectual disability and/or autism spectrum disorder. All pupils more likely followed requests by tactile symbols than when asked verbally. Tactile symbols seemed to be essential to increase the activity of pupils with the combination of vision impairment and mental disorder.

\section{Related Projects}

In this section, we list approaches that aim to support orientation in the space environment, spatial mental modeling or use a special method for sensory substitution.

Several commercially available applications aim to support navigation of those blind and visually impaired. BlindSquare ${ }^{1}$ is one of the most popular GPS navigation application globally. BlindSquare uses OpenStreetMaps [55] as source for geographical information and Foursquare $^{2}$ for information about points of interest.

Naviterier $^{3}$ is an emerging project currently available in the Czech Republic. Unlike other approaches, it utilizes detailed geographical information about a sidewalk network to provide the navigational information for the visually impaired. Another feature if NaviTerier is that it does not rely on positional information provided by global navigation satellite systems. The main reason is that the position accuracy in the city environment can be significantly decreased by the limited direct visibility of the sky and by signal reflections.

\footnotetext{
${ }^{1}$ http://blindsquare.com/

${ }^{2}$ https://foursquare.com

${ }^{3}$ https://naviterier.cz
} 
In [7] Balata et al. present a study investigating performance and issues of navigation of visually impaired individuals provided by another visually impaired utilizing teleassistance. Authors focused on problems in the navigator's attempts to direct the blind traveler to the destination. Most problems occurred during activities performed by the navigator.

Macik et al. [9] present an indoor navigation system for interiors adapted to support navigation needs of visually impaired. This system provides navigation cues to guide a blind traveler to the destination using navigation terminals embedded into a building interior. Authors argue that enhancing the indoor environment by means to support navigation and orientation is better than relying on the use of sophisticated equipment possessed by the user like contemporary smartphones. A qualitative study indicates that the system can effectively guide older adults and visually impaired to their destination along complex route in an indoor environment.

Zeng and Weber in [56] propose annotated interactive tactile maps for the visually impaired. It uses multi-line touch-sensitive braille display (array 60x120 pins) to convey geographic information while allowing the user to pan, zoom, and search but also create and share annotations about points of interest. Apart from touchsensitive braille display, the system consists of a GIS database and database of points of interest as well as from the annotation module, exploration module, and a presentation module. The interaction is based on tactile symbols that represent information through raised pins. The user can identify streets, buildings and various points of interest.

Albouys-Perrois et al. in [57] present a multisensory augmented reality (AR) map for blind and low vision individuals. Using participatory design (they collaborated with 15 visually impaired students and three orientation and mobility instructors) they developed a prototype that combines projection, audio output, and use of tactile tokens. The model allows both map exploration and constriction by low vision and blind people. The results show that models employing spatial augmented reality are useful mean for orientation and mobility training of visually impaired. Authors argue that such models should allow not only exploration but also map construction. Existing AR toolkits can be adapted to be used by visually impaired by adding audio and tactile cues.

Flores and Manduchi in [58] show an application for indoor backtracking assistance for the visually impaired. The system requires no maps of the building or environment modifications. The system records path from the starting location regarding a sequence of turns and step count. When requested, the system provides backtracking guidance by speech instruction about next turns and step count to follow. The system only measures right angle turns while assuming that most buildings have corridors intersecting at right angles.

Gollner et al. in [59] introduce a communication device for deaf-blind people. Lorm alphabet is a tactile hand-touch alphabet, where each character is assigned to 
a certain area of the hand. The Mobile Lorm Glove is communication and translation device that uses hand-touch alphabet Lorm to allow deaf-blind individuals to compose messages and to perceive the incoming messages. The glove uses pressure sensors to sense the user input and small vibration motors to provide tactile output. Unlike classical Lorm interaction, the Lorm Glove does not rely on physical contact, enables communication over distance, and one-to-many communication. Authors employed participatory design (involving target users in the design process) for the development.

In this section, we examined several practical commercial and research solutions that focus on navigation and spatial orientation of people with disabilities and selected methods for sensory substitution. It can be seen that many recent approaches reflect the user's cognitive properties either by addressing them during the development process but also by providing adaptations and adaptation while actually used. In the next section, we focus on discussion and recommendations for the design of CogInfoCom based solution to support orientation in space and spatial mental modeling.

\section{Conclusion and Future Research Direction}

In this paper, we discussed selected theoretical foundations for spatial orientation and spatial mental modeling within the framework of CogInfoCom. The future development in this domain can contribute to more efficient methods for supporting spatial mental modeling. By developing systems based on blended natural and artificial capabilities, we can facilitate orientation, navigation and spatial orientation training for individuals with different physical and cognitive capabilities.

Support of spatial mental modeling requires precise spatial information about the desired environment. Spatial information science [6] is now moving towards sharing spatial information for purposes and employing various cyber-physical systems. This new trend can bring substantial benefits also for support of mental spatial modeling in live cognitive entities. Up to date spatial information that is easier to get and maintain is vital for navigation and orientation support.

Identifying the relevant information for a particular task is essential for successful applications. According to [49], this task is often neglected by researchers. It is also necessary to find an efficient method of how to present the information to a particular user. Research of [49] shows properties and limitation of non-visual modalities usable for sensory substitution for purposes of those blind and visually impaired.

It is possible to use virtual reality for purposes of orientation and mobility training of visually impaired (e.g., [40]). It is an example of employing infocommunication technologies to facilitate spatial understanding. Vice-versa virtual reality environments should support easy and natural navigation methods while promoting natural spatial orientation and navigation. Albouys-Perrois et al. in [57] 
went even further and employed augmented reality to enable visually impaired to not only explore but also create multisensory maps.

Our research shows that personalization on an individual level might be necessary for providing a successful solution for a broader audience. The number of individuals affected by vision loss is growing despite new therapeutic options offered by medicine. Also, there is a high percentage of older adults that are challenged by vision impairment or vision loss. This group is also often affected by other age-related health issues, either physical or cognitive. Method for presentation spatial information should reflect individual needs and limitations. The emerging CogInfoCom technologies should enable utilizing blended natural and cognitive capabilities sensing personal characteristics and adapt the interaction accordingly.

We see the natural collaboration of interconnected natural and artificial cognitive entities as the logical next step in the interaction. Ultimately, an artificial cognitive entity would have to sense and understand cognition of a human individual - the way one thinks. This ultimate goal is not achievable in the full extent by means available nowadays. However, research and development in the field of CogInfoCom can narrow the gap to reach this goal.

Several papers in the domain of CogInfoCom focused on detection of various personal cognitive properties. Speech features have been used to estimate severity of Parkinson's disease [60], detect depression [61, 62], or elucidate on body condition [63]. Alam et al. in [64] focus on detection of empathy in human spoken conversation. Rusko and Finke [65] suggest to use speech analysis to improve the safety of air traffic management. Stress detection based on analysis of user diaries is described in [66]. Classification of cognitive workload using cardiovascular measures is investigated in [67]. In [68], Lewandowska et al. investigate culturespecific emotion models in human-robot interaction. Authors of [69] propose an automatic cognitive profiling system for an adaptive educational gaming platform.

\section{Acknowledgments}

This research has been supported by the Technology Agency of the Czech Republic under the research program TE01020415 (V3C - Visual Computing Competence Center) and by the project Navigation of handicapped people funded by grant no. SGS16/236/OHK3/3T/13 (FIS 161 - 1611663C000).

\section{References}

[1] Baranyi, P., Csapó, Á.: Definition and Synergies of Cognitive Infocommunications. Acta Polytechnica Hungarica, 9 (1), 2012, pp. 67-83

[2] Bouchard Ryan, E. et al.: Coping with Age-related Vision Loss in Everyday Reading Activities. Educational Gerontology, 29 (1), 2003, pp. 37-54

[3] Horowitz, A. et al.: The Impact of Assistive Device Use on Disability and Depression among Older Adults with Age-related Vision Impairments. The 
Journals of Gerontology Series B: Psychological Sciences and Social Sciences, 61 (5), 2006, pp. S274-S280

[4] Macik, M. et al.: How can ict help the visually impaired older adults in residential care institutions: The everyday needs survey. In: Cognitive infocommunications (coginfocom), $20178^{\text {th }}$ IEEE international conference on. IEEE, 2017, pp. 000157-000164

[5] Wobbrock, J. O. et al.: Ability-based Design: Concept, Principles and Examples. ACM Transactions on Accessible Computing (TACCESS) 3 (3) 2011, p. 9

[6] Um, J.-S.: Embracing Cyber-physical System as Cross-Platform to Enhance Fusion-Application Value of Spatial Information. Spatial Information Research, 25 (3), 2017, pp. 439-447

[7] Balata, J. et al.: Navigation Problems in Blind-to-Blind Pedestrians TeleAssistance Navigation. In: Human-computer interaction. Springer, 2015, pp. 89-109

[8] Riganova, M. et al.: Crowdsourcing of Accessibility Attributes on Sidewalkbased Geodatabase. In: IFIP conference on human-computer interaction. Springer, 2017, pp. 436-440

[9] Macik, M. et al.: Smartphoneless Context-Aware Indoor Navigation. In: Cognitive infocommunications (coginfocom), $20167^{\text {th }}$ IEEE international conference on. IEEE, 2016, pp. 000163-000168

[10] Palivcova, D. et al.: SuSy: Surveillance System for Hospitals. In: Cognitive infocommunications (coginfocom), $20178^{\text {th }}$ IEEE international conference on. IEEE, 2017, pp. 000131-000136

[11] Gintner, V. et al.: Improving Reverse Geocoding: Localization of Blind Pedestrians Using Conversational ui. In: Cognitive infocommunications (coginfocom), $20178^{\text {th }}$ IEEE international conference on. IEEE, 2017, pp. 000145-000150

[12] Ito, A. et al.: A Cognitive Model of Sightseeing for Mobile Support System. In: Cognitive infocommunications (coginfocom), $20178^{\text {th }}$ IEEE international conference on. IEEE, 2017, pp. 000057-000062

[13] Sik, D. et al.: Implementation of a Geographic Information System with Big Data Environment on Common Data Model. In: Cognitive infocommunications (coginfocom), $20178^{\text {th }}$ IEEE international conference on. IEEE, 2017, pp. 000181-000184

[14] Dictionaries, O.: Definition of Navigation in English. webpage, 2018

[15] Dictionaries, O.: Definition of Orientation in English. webpage, 2018

[16] Stanton, N. A. et al.: Situational Awareness and Safety. Safety science, 39 (3), 2001, pp. 189-204 
[17] Dictionaries, O.: Definition of Cognition in English. webpage, 2018

[18] Thagard, P.: Cognitive Science. In: The stanford encyclopedia of philosophy (Editor: E. N. Zalta).

https://plato.stanford.edu/archives/fall2014/entries/cognitive-science/;

Metaphysics Research Lab, Stanford University, 2014

[19] Chomsky, N.: Language and Mind. Cambridge University Press, 2006

[20] Kagan, J.: The concept of identification. Psychological review, 65 (5), 1958, p. 296

[21] Witkin, H. A. et al.: Field-Dependent and Field-Independent Cognitive Styles and Their Educational Implications. Review of educational research, 47 (1), 1977, pp. 1-64

[22] Richardson, A.: Verbalizer-Visualizer: A Cognitive Style Dimension. Journal of mental imagery, 1977

[23] Jung, C. G.: Psychological Types: Or the Psychology of Individuation. 1923

[24] Myers, I. B. et al.: Manual, a Guide to the Development and Use of the Myers-Briggs Type Indicator. Consulting Psychologists Press, 1985

[25] Gregorc, A. F.: Style as a Symptom: A Phenomenological Perspective. Theory into Practice, 23 (1), 1984, pp. 51-55

[26] Sternberg, R. J.: Mental Self-Government: A Theory of Intellectual Styles and Their Development. Human Development, 31 (4), 1988, pp. 197-224

[27] Sternberg, R. J., Grigorenko, E. L.: Are Cognitive Styles still in Style? American psychologist, 52 (7), 1997, p. 700

[28] Torok, A.: From Human-Computer Interaction to Cognitive Infocommunications: A Cognitive Science Perspective. In: Cognitive infocommunications (coginfocom), $20167^{\text {th }}$ IEEE international conference on. IEEE, 2016, pp. 000433-000438

[29] Ungar, S.: Cognitive Mapping without Visual Experience. Cognitive mapping: past, present, and future, 4, 2000, p. 221

[30] Gattis, M.: Spatial Schemas and Abstract Thought. MIT press, 2003

[31] Franc, J.: Psychologické aspekty navigace nevidomych. 2014

[32] Millar, S.: Understanding and Representing Space: Theory and Evidence from Studies with Blind and Sighted Children. Clarendon Press/Oxford University Press, 1994

[33] Thinus-Blanc, C., Gaunet, F.: Representation of Space in Blind Persons: Vision as a Spatial Sense? Psychological bulletin, 121 (1), 1997, p. 20 
[34] Loomis, J. M. et al.: Navigating without Vision: Basic and Applied Research. Optometry and Vision Science, 78 (5), 2001, pp. 282-289

[35] Kitchin, R. M. et al.: Understanding Spatial Concepts at the Geographic Scale without the Use of Vision. Progress in Human Geography, 21 (2), 1997, pp. 225-242

[36] Siegel, A. W., White, S. H.: The Development of Spatial Representations of Large-Scale Environments. In: Advances in child development and behavior. Elsevier, 1975, pp. 9-55

[37] Brock, A. M. et al.: Interactivity Improves Usability of Geographic Maps for Visually Impaired People. Human-Computer Interaction, 30 (2), 2015, pp. 156-194

[38] Loomis, J. M. et al.: Representing 3D Space in Working Memory: Spatial Images from Vision, Hearing, Touch, and Language. In: Multisensory imagery. Springer, 2013, pp. 131-155

[39] Lahav, O., Mioduser, D.: Construction of Cognitive Maps of Unknown Spaces Using a Multi-Sensory Virtual Environment for People Who are Blind. Computers in Human Behavior, 24 (3), 2008, pp. 1139-1155

[40] Lahav, O. et al.: Rehabilitation Program Integrating Virtual Environment to Improve Orientation and Mobility Skills for People Who are Blind. Computers \& education, 80, 2015, pp. 1-14

[41] Papadopoulos, K. et al.: Cognitive Maps of Individuals with Blindness for Familiar and Unfamiliar Spaces: Construction through Audio-Tactile Maps and Walked Experience. Computers in Human Behavior, 75, 2017, pp. 376384

[42] Kitchin, R., Jacobson, R. D.: Techniques to Collect and Analyze the Cognitive Map Knowledge of Persons with Visual Impairment or Blindness: Issues of validity. Journal of Visual Impairment and Blindness, 91 (4), 1997, pp. 360-376

[43] Kacorri, H. et al.: Environmental Factors in Indoor Navigation Based on Real-World Trajectories of Blind Users. In: Proceedings of the 2018 chi conference on human factors in computing systems. ACM, 2018, p. 56

[44] Izsó, L.: The Significance of Cognitive Infocommunications in Developing Assistive Technologies for People with Non-Standard Cognitive Characteristics: CogInfoCom for People with Non-Standard Cognitive Characteristics. In: Cognitive infocommunications (coginfocom), $20156^{\text {th }}$ IEEE international conference on. IEEE, 2015, pp. 77-82

[45] Esposito, A. et al.: Differences between Hearing and Deaf Subjects in Decoding Foreign Emotional Faces. In: Cognitive infocommunications (coginfocom), $20178^{\text {th }}$ IEEE international conference on. IEEE, 2017, pp. 000175-000180 
[46] Goldreich, D., Kanics, I. M.: Tactile Acuity is Enhanced in Blindness. Journal of Neuroscience, 23 (8), 2003, pp. 3439-3445

[47] Bavelier, D., Neville, H. J.: Cross-Modal Plasticity: Where and How? Nature Reviews Neuroscience, 3 (6), 2002, p. 443

[48] Millar, S.: Space and Sense: Essays in Cognitive Psychology. New York, NY: Psychology Press, 2008

[49] Loomis, J. M. et al.: Sensory Substitution of Vision: Importance of Perceptual and Cognitive Processing. Assistive technology for blindness and low vision, 2012, pp. 162-191

[50] Bennett, C. R. et al.: Spatial Updating of Multiple Targets: Comparison of Younger and Older Adults. Memory \& cognition, 45 (7), 2017, pp. 12401251

[51] Csapó, Á. et al.: A Survey on Hardware and Software Solutions for Multimodal Wearable Assistive Devices Targeting the Visually Impaired. Acta Polytechnica Hungarica, 13 (5), 2016, pp. 39-63

[52] Loomis, J. M., Lederman, S. J.: Tactual Perception. Handbook of Perception and Human Performances, 2, 1986, p. 2

[53] Holloway, L. et al.: Accessible Maps for the Blind: Comparing 3D Printed Models with Tactile Graphics. In: Proceedings of the 2018 chi conference on human factors in computing systems. ACM, 2018, p. 198

[54] Aasen, G., Nærland, T.: Enhancing Activity by Means of Tactile Symbols: A Study of a Heterogeneous Group of Pupils with Congenital Blindness, Intellectual Disability and Autism Spectrum Disorder. Journal of Intellectual Disabilities, 18 (1), 2014, pp. 61-75

[55] Haklay, M., Weber, P.: Openstreetmap: User-generated Street Maps. IEEE Pervasive Computing, 7 (4), 2008, pp. 12-18

[56] Zeng, L., Weber, G.: ATMap: Annotated Tactile Maps for the Visually Impaired. In: Cognitive behavioural systems. Springer, 2012, pp. 290-298

[57] Albouys-Perrois, J. et al.: Towards a Multisensory Augmented Reality Map for Blind and Low Vision People: A Participatory Design Approach. In: Proceedings of the 2018 chi conference on human factors in computing systems. ACM, 2018, p. 629

[58] Flores, G., Manduchi, R.: Easy Return: An App for Indoor Backtracking Assistance. In: Proceedings of the 2018 chi conference on human factors in computing systems. New York, NY, USA: ACM, 2018, pp. 17:1-17:12

[59] Gollner, U. et al.: Mobile Lorm Glove: Introducing a Communication Device for Deaf-Blind People. In: Proceedings of the sixth international conference on tangible, embedded and embodied interaction. ACM, 2012, pp. $127-130$ 
[60] Sztahó, D. et al.: Automatic Estimation of Severity of Parkinson's Disease Based on Speech Rhythm Related Features. In: Cognitive infocommunications (coginfocom), $20178^{\text {th }}$ IEEE international conference on. IEEE, 2017, pp. 000011-000016

[61] Kiss, G., Vicsi, K.: Investigation of Cross-Lingual Depression Prediction Possibilities Based on Speech Processing. In: Cognitive infocommunications (coginfocom), $20178^{\text {th }}$ IEEE international conference on. IEEE, 2017, pp. 000097-000102

[62] Kiss, G., Vicsi, K.: Comparison of Read and Spontaneous Speech in Case of Automatic Detection of Depression. In: Cognitive infocommunications (coginfocom), $20178^{\text {th }}$ IEEE international conference on. IEEE, 2017, pp. 000213-000218

[63] Kiss, G. et al.: Connection between Body Condition and Speech Parameters-Especially in the Case of Hypoxia. In: Cognitive infocommunications (coginfocom), $20145^{\text {th }}$ IEEE conference on. IEEE, 2014, pp. 333-336

[64] Alam, F. et al.: Can We Detect Speakers' Empathy?: A Real-Life Case Study. In: Cognitive infocommunications (coginfocom), $20167^{\text {th }}$ IEEE international conference on. IEEE, 2016, pp. 000059-000064

[65] Rusko, M., Finke, M.: Using Speech Analysis in Voice Communication: A New Approach to Improve Air Traffic Management Security. In: Cognitive infocommunications (coginfocom), $20167^{\text {th }}$ IEEE international conference on. IEEE, 2016, pp. 000181-000186

[66] Ghosh, A. et al.: Are You Stressed? Detecting High Stress from User Diaries. In: Cognitive infocommunications (coginfocom), $20178^{\text {th }}$ IEEE international conference on. IEEE, 2017, pp. 000265-000270

[67] Magnusdottir, E. H. et al.: Cognitive Workload Classification Using Cardiovascular Measures and Dynamic Features. In: Cognitive infocommunications (coginfocom), $20178^{\text {th }}$ IEEE international conference on. IEEE, 2017, pp. 000351-000356

[68] Lewandowska-Tomaszczyk, B., Wilson, P. A.: Compassion, Empathy and Sympathy Expression Features in Affective Robotics. In: Cognitive infocommunications (coginfocom), $20167^{\text {th }}$ IEEE international conference on. IEEE, 2016, pp. 000065-000070

[69] Pomázi, K. et al.: Self-Standardizing Cognitive Profile Based on Gardner's Multiple Intelligence Theory. In: Cognitive infocommunications (coginfocom), $20167^{\text {th }}$ IEEE international conference on. IEEE, 2016, pp. 000317-000322 